\title{
Processed Panax ginseng, Sun Ginseng Increases Type I Collagen by Regulating MMP-1 and TIMP-1 Expression in Human Dermal Fibroblasts
}

\author{
Kyu Choon Song ${ }^{1 \#}$, Tong-Shin Chang ${ }^{2 \#}$, Hyejin Lee ${ }^{1 \#}$, Jinhee Kim¹, Jeong Hill Park ${ }^{3}$, and Gwi Seo \\ Hwang $^{1 *}$ \\ ${ }^{1}$ Lab of Cell Differentiation Research, College of Oriental Medicine, Gachon University, Seongnam 461-701, Korea \\ ${ }^{2}$ Division of Life \& Pharmaceutical Sciences, Center for Cell Signaling \& Drug Discovery Research, College of Pharmacy, \\ Ewha Womans University, Seoul 120-750, Korea \\ ${ }^{3}$ College of Pharmacy, Seoul National University, Seoul 151-742, Korea
}

In the present study, effects of sun ginseng (SG) on the collagen synthesis and the proliferation of dermal fibroblast were investigated. Collagen synthesis was measured by assaying procollagen type I C-peptide production. In addition, the level of matrix metalloproteinase (MMP)-1 was assessed by western blot analysis. SG suppressed the MMP-1 protein level in a dose-dependent manner. In contrast, SG dose-dependently increased tissue inhibitors of MMP (TIMP)-1 production in fibroblasts. SG increased type I collagen production directly and/or indirectly by reducing MMP-1 and stimulating TIMP-1 production in human dermal fibroblasts. SG dose-dependently induced fibroblast proliferation and this, in turn, can trigger more collagen production. These results suggest that SG may be a potential pharmacological agent with anti-aging properties in cultured human skin fibroblast.

Keywords: Panax ginseng, Sun ginseng (SE), Type I collagen, Matrix metalloproteinase-1, Human skin fibroblasts

\section{INTRODUCTION}

The signs of aging skin are wrinkle formation, irregularity of pigmentation, dryness, red blotches, and decreasing elasticity. Cutaneous aging has two distinct processes. The first is intrinsic, or innate aging, while the second is extrinsic, also known as photoaging. In any process, there are notable changes in the dermis. The dermis is a deeper layer of human skin that supports the blood vessels and nerves while maintaining other functions. Major extracellular components of the dermis are the ground substance, collagen fibers, and elastins, all of which are important to maintain the physiological functions of the skin [1].

Collagens are extracellular matrix fibrillar molecules in dermal connective tissue and comprise about $80 \%$ of

(cc) This is an Open Access article distributed under the terms of the Creative Commons Attribution Non-Commercial License (http://creativecommons.org/licenses/by-nc/3.0/) which permits unrestricted non-commercial use, distribution, and reproduction in any medium, provided the original work is properly cited. the extracellular material, contributing to its strength and facilitating elasticity, tightening, and the cell integrity of the skin. More than 20 different subtypes of collagen have been identified and type I collagen is the predominant form in the human skin. It is synthesized by fibroblasts. Interstitial collagens are synthesized and secreted as procollagens. Procollagens are cleaved into collagen molecules, carboxy- and amino-terminal propeptides by specific proteases to form collagen peptides followed by formation of complete collagen fibers [2]. Therefore, the production of carboxy-terminal propeptide (procollagen C-peptide) represents the extent of collagen fiber synthesis. In aged skin, epidermis cell proliferation slows down and the dermis becomes were thinner with the reduction

\footnotetext{
Received 02 Dec. 2011, Revised 29 Dec. 2011, Accepted 29 Dec. 2011

\# These authors contributed equally to this work.

*Corresponding author

E-mail: seoul@kyungwon.ac.kr

Tel: +82-31-750-5421, Fax: +82-31-721-7029
} 
of the quantity of collagen.

Matrix metalloproteinases (MMPs) are a family of enzymes that are responsible for the degradation and modification of the extracellular matrix. MMPs can be divided into at least five subfamilies according to their substrate specificity, structure and cellular localization. Specifically, MMP-1 (collagenase-1) from fibroblasts preferentially initiates the degradation of fibrillar collagen molecules, leading to skin fragility, laxity, roughness, dryness, pigmentation, and wrinkle formation. An age-dependent increase of MMP-1 has been observed in the dermis due to a reduction in the tissue inhibitors of MMPs (TIMPs) [3].

TIMPs contain four different subtypes and are produced by many types of cells, including fibroblasts. A balance between MMPs and TIMPs is important to control Hortrol the tissue remodeling and degradation in the skin. Hornebeck [4] reported that TIMP expression decreases with senescence in fibroblasts and Koike et al. [5] reported that TIMP-2 modulates decreased angiogenesis in aged tissues via the TIMP-2-mediated inhibition of MMP-2. These investigations suggest that the prevention of collagen degradation by MMP and the inhibiton of MMPs by TIMP should be considered as part of an antiaging treatment for skin.

Panax ginseng is a widely used herb in Asian countries, having anti-inflammatory, antioxidant, anti-tumor promoting and anti-aging potencies. There are several types of ginseng depending on the processing methods, including fresh ginseng, white ginseng (WG) and red ginseng $(\mathrm{RG})$. Fresh ginseng is intact and undried ginseng root, while air-dried ginseng root is referred to as WG. RG is a type of steamed $\left(98^{\circ} \mathrm{C}\right.$ to $\left.100^{\circ} \mathrm{C}\right)$ and dried ginseng root. $\mathrm{RG}$ acquires unique less-polar ginsenosides during the steaming process [6], but the amount is very small. Cho et al. [7] reported a new processed ginseng, named as 'sun ginseng (SG)', which was prepared by steaming at higher temperature to increase the content of less-polar ginsenosides of $\mathrm{Rg}_{3}, \mathrm{Rg}_{5}$ and $\mathrm{Rk}_{1}$, consequently improving its therapeutic efficacy. Increases in its anticarcinogenic activity [8], free radical scavenging activity $[9,10]$, nephroprotective activity $[11,12]$, and anti-platelet activity [13] have been reported.

In the present study, changes in the production of procollagen type I C-peptide (PICP) and the protein level of MMP-1 in fibroblasts after SG treatment were investigated. Also, the mRNA level of TIMP-1 was measured to verify the correlation between MMP-1 and TIMP1 expression. The results show that SE increased type I collagen production by reducing MMP-1 and stimulating
TIMP-1 production in human dermal fibroblast.

\section{MATERIALS AND METHODS}

\section{Reagents}

3-(4,5-dimethylthiazol-2-yl)-2,5-diphenyltetrazolium bromide (MTT) and lactate dehydrogenase (LDH) assay kits were purchased from Sigma (St. Louis, MO, USA). The human TIMP-1 immunoassay kit was purchased from R\&D Systems (Minneapolis, MN, USA). The PICP immunoassay kit was obtained from Takara Bio Inc. (Otsu, Japan). Polyclonal antibody against human MMP1 was obtained from EMD Millipore (Calbiochem, CA, USA), and secondary horseradish peroxidase (HRP)conjugated anti-rabbit IgG was purchased from Santa Cruz Biotechnology Inc. (Santa Cruz, CA, USA). WG and SG extracts were provided by Ginseng Science Inc. (Seoul, Korea).

\section{Cell culture and treatment}

Human dermal fibroblasts purchased from Lonza (Walkersville, MD, USA) were maintained at $37^{\circ} \mathrm{C}$ in Dulbecco's modified Eagle's medium (DMEM) (WelGENE, Daegu, Korea) supplemented with 10\% heatinactivated fetal bovine serum (FBS) (Lonza) and 1\% penicillin/streptomycin (Gibco BRL Life Technology, Grand Island, NY, USA) in a 5\% $\mathrm{CO}_{2}$-air atmosphere. To investigate the effect of ginseng, dermal fibroblasts were stabilized for $6 \mathrm{~h}$ and treated with different concentrations of ginseng for $3 \mathrm{~d}$.

\section{Cell proliferation assay}

To measure the effect of ginseng on dermal fibroblast proliferation, cell counting was performed. Dermal fibroblasts at $2 \times 10^{4}$ cells were plated into 6-well plates and incubated for $3 \mathrm{~d}$ in DMEM containing 5\% FBS in the presence or absence of ginseng. Following incubation, the cells were trypsinized, washed with phosphate buffered saline, and stained by trypan blue. The viable cells were counted in a phase-contrast microscope using a hemocytometer.

\section{Procollagen type I C-peptide and tissue inhibitors of matrix metalloproteinase-1 assay}

To assess the effect of ginseng on the PICP and TIMP1 release from dermal fibroblasts, cells were treated and incubated as mentioned above. For the PICP assay, the conditioned media were transferred into new plate and measured with a PICP sandwich immunoassay kit according to the manufacturer's instructions. TIMP-1 
secretion level in the conditioned media was also measured using a TIMP-1 immunoassay kit. The assay was performed by the manufacturer's instruction.

\section{Preparation of secreted proteins and Western blot analysis}

To assess the influence of ginseng on the levels of MMP protein in conditioned media, a western blot analysis was performed. Dermal fibroblasts were seeded onto 6-well plates and incubated for 3 days in DMEM medium containing $5 \% \mathrm{FBS}$ and ginseng $(0,2,10$, or $50 \mu \mathrm{g} /$ $\mathrm{mL})$. The conditioned media were collected and mixed with $15 \%$ trichloroacetic acid. The proteins in the media were precipitated by centrifugation $(8,000 \mathrm{rpm}$ for 10 $\min$ at $4{ }^{\circ} \mathrm{C}$ ) and washed with acetone. The precipitated proteins were dissolved in a buffer $(25 \mathrm{mM}$ Tris- $\mathrm{Cl} \mathrm{pH}$ $7.5,100 \mathrm{mM} \mathrm{NaCl}, 1 \% \mathrm{NP}-40,1 \%$ sodium deoxycholate, $0.1 \%$ sodium dodecyl sulfate (SDS) and protease inhibitor cocktails (Roche Applied Science, Mannheim, Germany) and quantified with a BCA assay using a Pierce BCA protein assay kit (Pierce, Rockford, IL, USA). Twenty five $\mu \mathrm{g}$ of proteins were loaded onto SDSpolyacrylamide gel, electrophoresed, and transferred to a PVDF membrane. The membrane was probed with polyclonal anti-MMP1 $(1 \mu \mathrm{g} / \mathrm{mL})$ and a HRP-conjugated second antibody. MMP-1 proteins were visualized with the ECL system from Bio-Rad (Hercules, CA, USA).

\section{Statistical analysis}

All experiments were repeated at least three times, and the data are presented as means $\pm \mathrm{SD}$. of triplicate determinations from representative experiments. Statistical analyses were performed using Student's $t$-test. Differences were considered significant at ${ }^{*} p<0.05$ and ${ }^{* *} p<0.01$ vs. normal control (NC).

\section{RESULTS AND DISCUSSION}

\section{Sun ginseng increases cell proliferation of skin fi- broblasts}

As aging progresses, the cell number in dermal fibroblasts decreases and the function of fibroblasts supporting healthy skin declines. Fibroblasts are the principle cellular component in the dermis layer of the skin, playing a role in the organization and remodeling of the extracellular matrix (ECM) in connective tissues by the production of collagen, fibronectins and elastins. Fibroblasts communicate with different cells in the skin through autocrine or paracrine signaling or by other methods. Reduction in the number and function of fi-

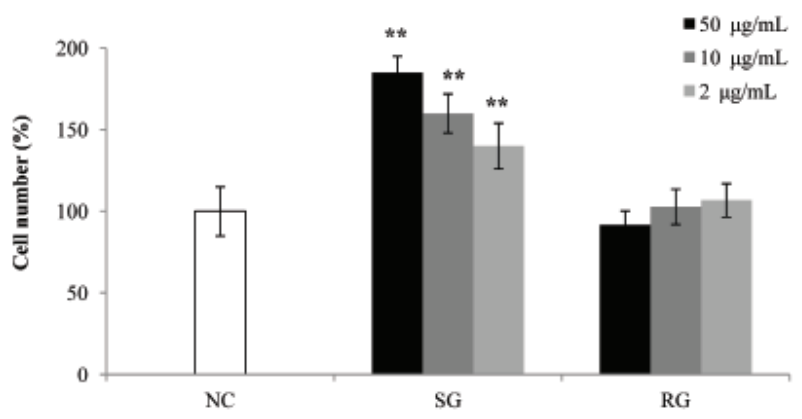

Fig. 1. Effects of ginseng extracts on cell proliferation of dermal fibroblasts. To measure the effect of the sun ginseng (SG) and red ginseng (RG) extracts on cell proliferation, dermal fibroblasts were plated into 6-well plates and incubated for $3 \mathrm{~d}$ in the presence or absence of ginseng extracts $(2,10$, and $50 \mu \mathrm{g} / \mathrm{mL})$. Following incubation, cells were stained by trypan blue and were counted using a hemocytometer. Cell viability was expressed as a percentage of an untreated group (normal control, NC). Data represent the mean $\pm S D$ of triplicate experiments. Differences were considered significant at $" p<0.01$ vs. NC.

broblasts subsequently cause a decrease of the collagen content in skin and thus lead to signs of skin aging, such as skin thinning, wrinkles, and a decline in elasticity. In previous studies, natural compounds which increase fibroblast proliferation were used as potential agents for a treatment against skin aging. Fucose-rich oligo and polysaccharides obtained from bacterial polysaccharides can slow the cellular and molecular skin aging mechanism in cultured human skin fibroblasts [14]. Phillips et al. [15] suggested the possibility of ascorbic acid as a growth factor that works by stimulating newborn and elderly cells to proliferate.

To investigate the effect of the ginseng extracts on dermal fibroblast proliferation, dermal fibroblasts were exposed dose-dependently to each of the ginseng extracts. To assess the cell proliferation state, viable cells were counted after trypan blue exclusion using a hemocytometer and the morphological change in the fibroblasts was observed. To compare the effect of SG with that of RG, the fibroblasts were treated with RG and SG extracts (2, 10 , and $50 \mu \mathrm{g} / \mathrm{mL}$ ) as shown in Fig. 1. Kim et al. reported that topical application of the total saponin fraction of red ginseng inhibits ultraviolet B-induced skin aging phenotypes (skin thickness, wrinkle formation and a reduction in skin elasticity) in hairless mice [16] and C57BL mice [17]. Moreover, it has been demonstrated that $10 \mu \mathrm{g} / \mathrm{m}$ of RG extract increases the collagen synthesis and inhibits the MMP-1 activity in human dermal fibroblast, while decreases the cell proliferation by $16 \%$ [18].

In the present study, the RG treatments did not show significant change in dermal fibroblast proliferation as compared with NC (Fig. 1). On the other hand, SG treat- 


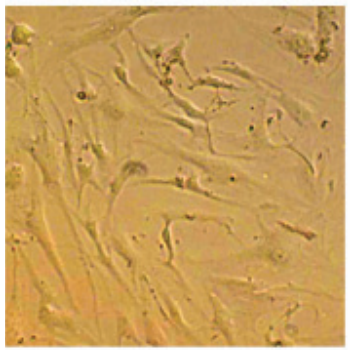

Normal control

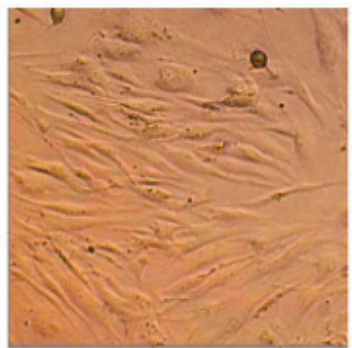

Sun ginseng

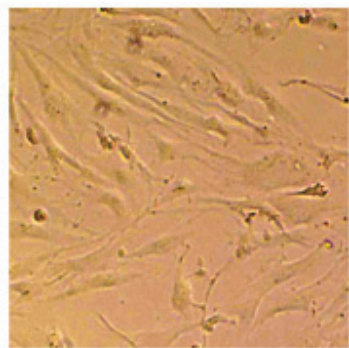

White ginseng

Fig. 2. Images of dermal fibroblasts cultured with various ginseng extracts. Dermal fibroblasts were plated onto 6-well plates and incubated for 3 $\mathrm{d}$ in the presence or absence of $50 \mu \mathrm{g} / \mathrm{mL}$ of each ginseng extract. The images of the cultured fibroblasts were obtained using an inverted microscope at $\times 100$ magnification.

ment dose-dependently increased cell numbers in the dermal fibroblasts. 2,10 and $50 \mu \mathrm{g} / \mathrm{mL}$ of SG resulted in a $40 \%, 60 \%$, and $85 \%$ increase in the cell numbers respectively as compared to NC. To verify the effect of SG on the proliferation of human dermal fibroblast, cells were cultured with $50 \mu \mathrm{g} / \mathrm{mL}$ of RG and SG extracts and photographed (Fig. 2). Dermal fibroblasts treated with SG maintained the fibroblastic shape and higher density indicating higher rate of proliferation as compared to NC.

\section{Sun ginseng increases procollagen type I C-pep- tide production in skin fibroblasts}

One of the significant alterations of connective tissue during the skin aging progress is the decrease of ECM production. The major component of ECM (more than $80 \%$ ) is collagen produced by fibroblast. Type I collagen comprises $85 \%$ of the total collagen. It is composed of a heterotrimer of two $\alpha 1$ (I) chains and one $\alpha 2$ (I) chain. Interstitial collagen molecules are synthesized as procollagens and then undergo various post-translational processes, including heterotrimer propeptide cleavage. As pro-collagen type I amino- and carboxy terminal propeptide (procollagen type I amino propeptide and PICP) are cleaved from pro-collagen molecules for mature collagen fibers, the amount of secreted PICP in culture medium allows us to measure the collagen synthesis [19]. A PICP radioimmunoassay kit is commercially available. In our experiments, SG increased the PICP release into the media up to $32 \%$ as compared to NC (Fig. 3).

The fibroblasts with aged phenotypes became wide and flat with irregular shapes and had lobulated nuclei that could not proliferate and synthesize collagen. In aged human skin, the type I collagen content decreased in vivo and in vitro as compared to young fibroblasts [20]. According to recent studies, the extracts from various types of ginseng modified by different processes increased type I procollagen production through Smad signaling activa-

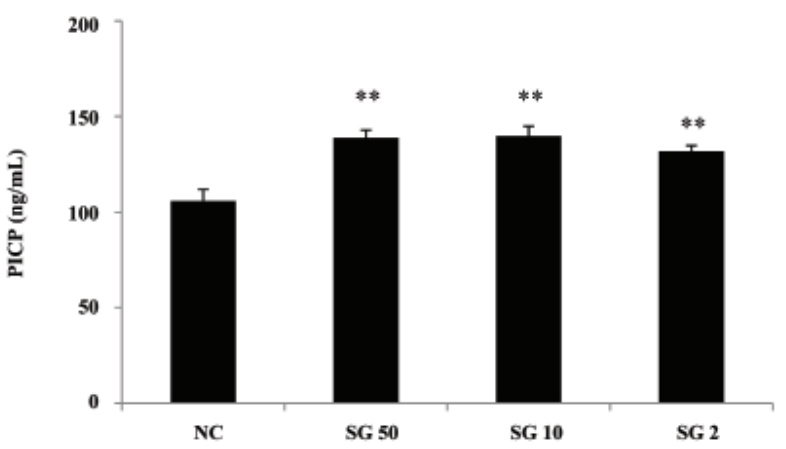

Fig. 3. Effects of sun ginseng (SG) on procollagen type I C-peptide (PICP) production on dermal fibroblasts. PICP production was measured with a PICP sandwich immunoassay kit according to the manufacturer's instructions using the conditioned media. Dermal fibroblasts were treated and incubated as mentioned in the text. Data represent the mean $+S D$ of triplicate experiments. NC, normal control; SG $50, S G 10$, and SG 2, indicate that cells were treated with 50,10 , and $2 \mu \mathrm{g} / \mathrm{mL}$ of SG, respectively. Differences were considered significant at " $p<0.01$ vs. NC.

tion [21] or MMP activation [18] in human dermal fibroblasts. In our study, SG effectively increased endogenous collagen synthesis in dermal fibroblasts with lower doses as compared to those used in other studies [18,21]. As the newly synthesized collagen plays a role in stimulating the fibroblast proliferation, the increased number of fibroblasts may produce more collagen molecules. This implies that SG treatment can delay skin aging processes through stimulating the fibroblast proliferation, which in turn causes an increased collagen synthesis in fibroblasts.

\section{Sun ginseng alters TIMP-1 production and MMP-1 expression in skin fibroblasts}

Remodeling of ECM by fibroblasts is regulated by MMPs. Several types of MMPs are responsible for degradation and modification of the extracellular matrix fibrilar molecules, collagens and elastins in the dermis. Particularly, MMP-1 (collagenase-1) initiates the degradation of type I collagen fibers and produces collagen 
peptides. These denatured collagens are more susceptible to other proteolytic enzymes. It has been reported that high levels of MMP expression and collagenase activity was observed in aged fibroblasts [22]. According to recent studies, procollagenase synthesis and collagenase activity are significantly high in the cultures of latepassage and Werner syndrome fibroblasts as compared to early-passage fibroblasts [23]. The increased MMP activity leads to a decrease in the collagen content in connective tissues and subsequently causes skin disease and an acceleration of aging signs. The tissue inhibitor of MMP, TIMP regulates MMP activity in the extracellular or pericellular space. As the balance between MMP-1 and TIMP-1 contributes to maintain the collagen content in normal healthy skin, the increased ratio of MMPs to TIMPs is well correlated with aging processes. The amount of MMP mRNA increases in senescent cell cultures. But, TIMP-1 mRNA levels are lower than those in early-passage cell cultures [3].

In this study, it was expected that SG would induce collagen synthesis by adjusting low MMP-1/TIMP-1 ratio. To evaluate the effect of SG on TIMP-1 production, the amount of TIMP-1 secretion was measured using a TIMP-1 immunoassay kit. As shown in Fig. 4, SG dosedependently increased TIMP-1 secretion into the culture media about $50 \%$ in SG $50 \mu \mathrm{g} / \mathrm{mL}$ as compared to untreated cells. Contrary to the increase in TIMP-1 production, the level of MMP-1 expression was significantly reduced by a SG treatment. A western blot analysis demonstrated that $\mathrm{SG}$ at dose of $50 \mu \mathrm{g} / \mathrm{mL}$ strongly reduced the MMP-1 expression in fibroblasts (Fig. 5).

Fujii et al. [24] reported that a plant extract from alma (Emblica officinalis Gaertn.) up-regulates procollagen production and TIMP-1 production in a manner that coincides with a decrease in MMP expression in human skin fibroblasts. An extract from Lupinus albus regulates the balance between MMPs and TIMPs in cultured human gingival fibroblasts [25]. Our data suggest that SG may protect dermal fibroblasts against aging by suppressing MMP expression and augmenting TIMP production, which is followed by the stimulation of collagen synthesis.

It has been well demonstrated that total extracts or purified ginsenosides from ginseng may protect human keratinocytes and fibroblasts from photodamage. Ginseng is a natural agent that is capable of modulating the cellular signaling involved in the UV-induced cell death and/or carcinogenesis [26,27]. In addition, Korean red ginseng extract shows anti-inflammatory and anti-oxidative effects in human keratinocytes [28]. Taken together, application of SG may be a potential trial to protect human skin against aging processes.

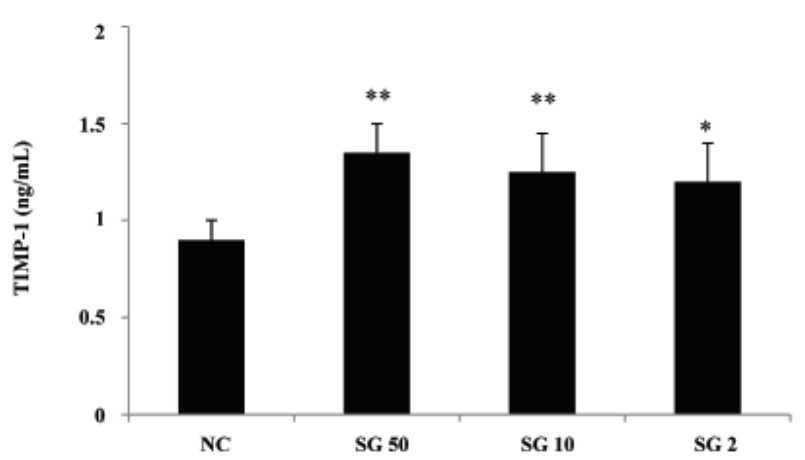

Fig. 4. Effects of sun ginseng (SG) on tissue inhibitors of matrix metalloproteinase (TIMP)-1 production in dermal fibroblasts. To determine the effects of SG, a TIMP-1 immunoassay was performed with conditioned media by the manufacture's instruction. Dermal fibroblasts were treated and incubated as mentioned in the materials and method. Data represent the mean $\pm S D$ of triplicate experiments. NC, normal control; SG 50, SG 10, and SG 2, SG 2 indicate that cells were treated with 50,10 , and $2 \mu \mathrm{g} / \mathrm{mL}$ of $S G$, respectively. 50 , 10 , and $2 \mu \mathrm{g} / \mathrm{mL}$ of $\mathrm{SG}$. Differences were considered significant at " $p<0.05$ and ${ }^{* *} p<0.01$ vs. NC.

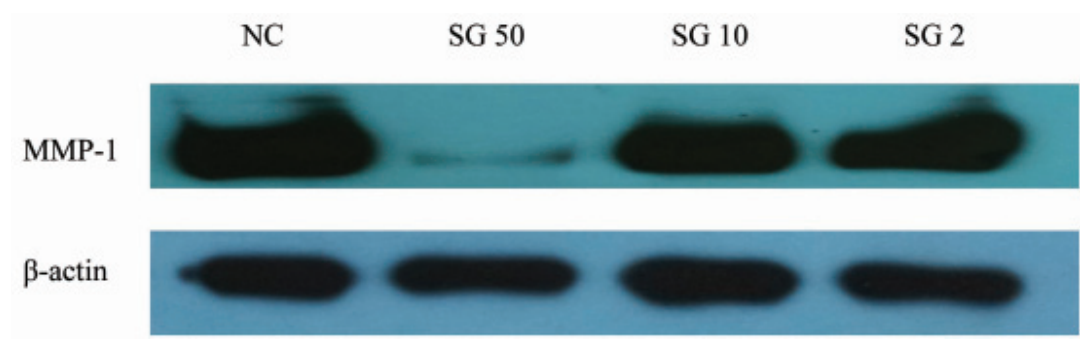

Fig. 5. Effects of sun ginseng (SG) on matrix metalloproteinase (MMP)-1 expression in dermal fibroblasts. Human dermal fibroblasts were seeded onto 6-well plates and incubated for $3 \mathrm{~d}$ in Dulbecco's modified Eagle's medium containing $5 \%$ fetal bovine serum and SG (2, 10, or $50 \mu \mathrm{g} /$ $\mathrm{mL}$ ). The protein in the media was concentrated with trichloroacetic acid solution and precipitated by centrifugation. Twenty five $\mu \mathrm{g}$ of protein was subjected to electrophoresis and a western blot analysis with polyclonal anti-MMP-1. Actin was used as a loading control. NC, normal control; SG 50 , SG 10, and SG 2, SG 2 indicate that cells were treated with 50,10, and $2 \mu \mathrm{g} / \mathrm{mL}$ of SG, respectively. with 50,10 , and $2 \mu \mathrm{g} / \mathrm{mL}$ of SG. 


\section{ACKNOWLEDGEMENTS}

This work was supported by Gachon University Research Fund and partly supported by Bio R\&D Program Grant M10642040002-07N4204-00210 and National Core Research Center Program Grant 2011-0006244 through the National Research Foundation of Korea funded by the Korea government (MEST) to Tong-Shin Chang

\section{REFERENCES}

1. Calleja-Agius J, Muscat-Baron Y, Brincat MP. Skin ageing. Menopause Int 2007;13:60-64.

2. Gordon MK, Hahn RA. Collagens. Cell Tissue Res 2010;339:247-257.

3. Millis AJ, Hoyle M, McCue HM, Martini H. Differential expression of metalloproteinase and tissue inhibitor of metalloproteinase genes in aged human fibroblasts. Exp Cell Res 1992;201:373-379.

4. Hornebeck W. Down-regulation of tissue inhibitor of matrix metalloprotease-1 (TIMP-1) in aged human skin contributes to matrix degradation and impaired cell growth and survival. Pathol Biol (Paris) 2003;51:569-573.

5. Koike T, Vernon RB, Gooden MD, Sadoun E, Reed MJ. Inhibited angiogenesis in aging: a role for TIMP-2. J Gerontol A Biol Sci Med Sci 2003;58:B798-B805.

6. Kim WY, Kim JM, Han SB, Lee SK, Kim ND, Park MK, Kim CK, Park JH. Steaming of ginseng at high temperature enhances biological activity. J Nat Prod 2000;63:1702-1704.

7. Cho S, Won CH, Lee DH, Lee MJ, Lee S, So SH, Lee SK, Koo BS, Kim NM, Chung JH. Red ginseng root extract mixed with Torilus fructus and Corni fructus improves facial wrinkles and increases type I procollagen synthesis in human skin: a randomized, double-blind, placebocontrolled study. J Med Food 2009;12:1252-1259.

8. Park IH, Piao LZ, Kwon SW, Lee YJ, Cho SY, Park MK, Park JH. Cytotoxic dammarane glycosides from processed ginseng. Chem Pharm Bull (Tokyo) 2002;50:538540.

9. Kang KS, Yokozawa T, Kim HY, Park JH. Study on the nitric oxide scavenging effects of ginseng and its compounds. J Agric Food Chem 2006;54:2558-2562.

10. Cho EJ, Piao KL, Jang MH, Baek SH, Kim HY, Kang KS, Kwon SW, Park JH. The effect of steaming on the free amino acid contents and antioxidant activity of Panax ginseng. Food Chem 2008;107:876-882.

11. Baek SH, Piao XL, Lee UJ, Kim HY, Park JH. Reduction of Cisplatin-induced nephrotoxicity by ginsenosides isolated from processed ginseng in cultured renal tubular cells. Biol Pharm Bull 2006;29:2051-2055.

12. Kang KS, Yamabe N, Kim HY, Park JH, Yokozawa T. Effects of heat-processed ginseng and its active component ginsenoside $20(\mathrm{~S})-\mathrm{Rg}_{3}$ on the progression of renal damage and dysfunction in type 2 diabetic Otsuka Long-Evans Tokushima Fatty rats. Biol Pharm Bull 2010;33:10771081.

13. Lee JG, Lee YY, Kim SY, Pyo JS, Yun-Choi HS, Park JH. Platelet antiaggregating activity of ginsenosides isolated from processed ginseng. Pharmazie 2009;64:602-604.

14. Peterszegi G, Isnard N, Robert AM, Robert L. Studies on skin aging. Preparation and properties of fucose-rich oligo- and polysaccharides. Effect on fibroblast proliferation and survival. Biomed Pharmacother 2003;57:187-194.

15. Phillips CL, Combs SB, Pinnell SR. Effects of ascorbic acid on proliferation and collagen synthesis in relation to the donor age of human dermal fibroblasts. J Invest Dermatol 1994;103:228-232.

16. Kim YG, Sumiyoshi M, Sakanaka M, Kimura Y. Effects of ginseng saponins isolated from red ginseng on ultraviolet B-induced skin aging in hairless mice. Eur J Pharmacol 2009;602:148-156.

17. Kim YG, Sumiyoshi M, Kawahira K, Sakanaka M, Kimura Y. Effects of red rinsing extract on ultraviolet Birradiated skin change in C57BL mice. Phytother Res 2008;22:1423-1427.

18. Kim N, Koo B, Lee S, Hwang E, So S, Do J. Effect of Korean red ginseng on collagen biosynthesis and MMPI activity in human dermal fibroblast. J Ginseng Res 2007;31;86-92.

19. Parfitt AM, Simon LS, Villanueva AR, Krane SM. Procollagen type I carboxy-terminal extension peptide in serum as a marker of collagen biosynthesis in bone. Correlation with Iliac bone formation rates and comparison with total alkaline phosphatase. J Bone Miner Res 1987;2:427-436.

20. Khorramizadeh MR, Tredget EE, Telasky C, Shen Q, Ghahary A. Aging differentially modulates the expression of collagen and collagenase in dermal fibroblasts. Mol Cell Biochem 1999;194:99-108.

21. Lee J, Jung E, Lee J, Huh S, Kim J, Park M, So J, Ham Y, Jung K, Hyun CG et al. Panax ginseng induces human Type I collagen synthesis through activation of Smad signaling. J Ethnopharmacol 2007;109:29-34.

22. Philips N, Smith J, Keller T, Gonzalez S. Predominant effects of Polypodium leucotomos on membrane integrity, lipid peroxidation, and expression of elastin and matrixmetalloproteinase-1 in ultraviolet radiation exposed fibroblasts, and keratinocytes. J Dermatol Sci 2003;32:1-9.

23. Wilhelm SM, Collier IE, Kronberger A, Eisen AZ, Marm- 
er BL, Grant GA, Bauer EA, Goldberg GI. Human skin fibroblast stromelysin: structure, glycosylation, substrate specificity, and differential expression in normal and tumorigenic cells. Proc Natl Acad Sci U S A 1987;84:67256729.

24. Fujii T, Wakaizumi M, Ikami T, Saito M. Amla (Emblica officinalis Gaertn.) extract promotes procollagen production and inhibits matrix metalloproteinase-1 in human skin fibroblasts. J Ethnopharmacol 2008;119:53-57.

25. Gaultier F, Foucault-Bertaud A, Lamy E, Ejeil AL, Dridi SM, Piccardi N, Piccirilli A, Msika P, Godeau G, Gogly B. Effects of a vegetable extract from Lupinus albus (LU105) on the production of matrix metalloproteinases (MMP1, MMP2, MMP9) and tissue inhibitor of metalloproteinases (TIMP1, TIMP2) by human gingival fibroblasts in cul- ture. Clin Oral Investig 2003;7:198-205.

26. Lee EH, Cho SY, Kim SJ, Shin ES, Chang HK, Kim DH, Yeom MH, Woe KS, Lee J, Sim YC et al. Ginsenoside F1 protects human $\mathrm{HaCaT}$ keratinocytes from ultraviolet-Binduced apoptosis by maintaining constant levels of Bcl2. J Invest Dermatol 2003;121:607-613.

27. Wang XY, Wang YG, Wang YF. Ginsenoside $R b_{1}, R_{1}$ and three extracts of traditional Chinese medicine attenuate ultraviolet B-induced G1 growth arrest in $\mathrm{HaCaT}$ cells and dermal fibroblasts involve down-regulating the expression of p16, p21 and p53. Photodermatol Photoimmunol Photomed 2011;27:203-212.

28. Hong CE, Lyu SY. Anti-inflammatory and Anti-oxidative effects of Korean red ginseng extract in human keratinocytes. Immune Netw 2011;11:42-49. 